\title{
AVALIAÇÃO QUALITATIVA DA INGESTÃO ALIMENTAR DE IDOSOS
}

Fernanda M. Pagotto (IC), Thiago M. F. de Souza (PG), Ivan L. P. Bonfante (PG), Leonardo A. de Lucena (IC), Maria L. Bellotto (PQ), Mara P. T. Chacon-Mikahil (PQ), Cláudia R. Cavaglieri (PQ)

\section{Resumo}

O processo de envelhecimento é comumente acompanhado pela diminuição da massa magra, da capacidade funcional, e o aumento da gordura corporal. O tecido adiposo em excesso é responsável pela liberação de uma variedade de adipocinas inflamatórias que contribuem para a inflamação sistêmica e desenvolvimento de DCNT's. A quantidade e o tipo do alimento ingerido estão altamente relacionados com os biomarcadores da inflamação sistêmica, assim é importante avaliar como está o hábito de ingestão alimentar em idosos.

Palavras Chave: Idoso, Hábito Alimentar, Inflamação

\section{Introdução}

O processo de envelhecimento causa diversos prejuízos fisiológicos, como o aumento de gordura visceral no indivíduo. O tecido adiposo em excesso contribui para a inflamação sistêmica e o consequente desenvolvimento de Doenças Crônicas Não Transmissíveis (DCNT's). Sabe-se que práticas alimentares saudáveis podem ajudar no tratamento e prevenção de DCNT's, devido ao papel modulador da alimentação sobre os biomarcadores da inflamação sistêmica. Assim, o conhecimento da ingestão de macro e micronutrientes em idosos pode fornecer importantes informações para a prevenção e/ou propensão destes indivíduos desenvolverem DCNT's. Com isso, o objetivo desse trabalho foi identificar a ingestão alimentar de macro e micronutrientes pró e anti-inflamatórios em idosos.

\section{Resultados e Discussão}

Foram selecionados 61 voluntários idosos ( 30 do sexo feminino e 31 do sexo masculino), que não apresentavam DCNT's, e que foram recrutados e orientados a preencher registros alimentares (RA's) de três dias, sendo pelo menos um dia do final de semana. Estes RA's foram avaliados quantitativamente, através do software de avaliação nutricional e prescrição dietética Dietpro® $5 \mathrm{i}$, tendo como base de dados à tabela Brasileira de Composição dos Alimentos (TACO) da UNICAMP. As informações obtidas desta análise foram contrastadas com as diretrizes de recomendação nutricional DRI's (2011). Os dados apresentados na tabela 1 mostram que os idosos estão propícios a uma dieta pró-inflamatória, por apresentarem principalmente: a ingestão de gordura saturada maior que a poli-insaturada e alto consumo de colesterol, além de baixo consumo de fibras alimentares e de vitamina $A$, que funcionam como anti-inflamatórios.
Tabela 1. Distribuição de macro e micronutrientes dos voluntários e valores referenciais.

\begin{tabular}{|c|c|c|c|}
\hline Nutrientes & Média & $\begin{array}{l}\text { VET } \\
(\%)\end{array}$ & Recomendação \\
\hline Carboidrato (g) & $255,4 \pm 95,8$ & 49,7 & $45-65 \%$ (VET) \\
\hline Proteína (g) & $93,9 \pm 32,7^{*}$ & 18,3 & $10-15 \%$ (VET) \\
\hline Lipídios (g) & $72,7 \pm 32,1$ & 31,9 & $20-35 \%$ (VET) \\
\hline G. Saturada (g) & $21,9 \pm 9,0$ & 5,2 & $7 \%$ (VET) \\
\hline G. Mono. (g) & $22,0 \pm 9,2$ & 5,2 & $10 \%(\mathrm{VET})$ \\
\hline G. Poli. (g) & $14,76 \pm 4,58$ & 3,49 & 10\% (VET) \\
\hline Fibras (g) & $23,62 \pm 8,46^{*}$ & - & $30(\mathrm{~g})$ \\
\hline Colesterol (mg) & $264,8 \pm 124,0^{*}$ & - & $300(\mathrm{mg})$ \\
\hline Sódio (mg) & $4206,4 \pm 1600,9^{*}$ & - & $1200(\mathrm{mg})$ \\
\hline Cálcio (mg) & $644,49 \pm 269,24^{*}$ & - & 1200 (mg) \\
\hline Ferro (mg) & $9,73 \pm 3,54$ & - & $8(\mathrm{mg})$ \\
\hline Vit. A ( $\mu \mathrm{g})$ & $191,52 \pm 114,85^{\star}$ & - & $900(\mu \mathrm{g})$ \\
\hline Vit. B1 (mg) & $1,28 \pm 0,59$ & - & $1,2(\mathrm{mg})$ \\
\hline Vit. B2 (mg) & $1,23 \pm 0,47^{*}$ & - & 1,3 (mg) \\
\hline Vit. B3 (mg) & $18,95 \pm 10,06$ & - & $16(\mathrm{mg})$ \\
\hline Vit. B6 (mg) & $1,06 \pm 0,50^{*}$ & - & 1,7 (mg) \\
\hline Vit. C (mg) & $135,54 \pm 93,52^{*}$ & - & $90(\mathrm{mg})$ \\
\hline
\end{tabular}

Legenda: $\mathrm{VET}=$ Valor energético total; $\mathrm{G}$. Saturada $=$ gordura saturada; G. Mono.= gordura monosaturada; G. Poli.= Gordura Poli-insaturada; Vit. = Vitamina. Valores em médias e desvio padrão. ${ }^{*}=$ média do grupo fora dos valores recomendados

\section{Conclusões}

Pode-se concluir que os idosos apresentam uma dieta com tendências pró-inflamatória, podendo colaborar para o desenvolvimento de DCNT's.

\section{Agradecimentos}

Conselho Nacional de Desenvolvimento Científico e Tecnológico (CNPQ)

${ }^{1}$ INSTITUTE OF MEDICINE. Dietary reference intakes. Tables of DRI values. Washington (DC): National Academy Press; 2011. [acesso 24 Jun 2015]. Disponível em: http://iom.edu/Activities/Nutrition/SummaryDRIs/DRI-Tables.aspx ${ }^{2}$ DE ESTUDOS, Núcleo. Tabela brasileira de composição de alimentos. In:Tabela brasileira de composição de alimentos. NEPA-UNICAMP, 2004. 